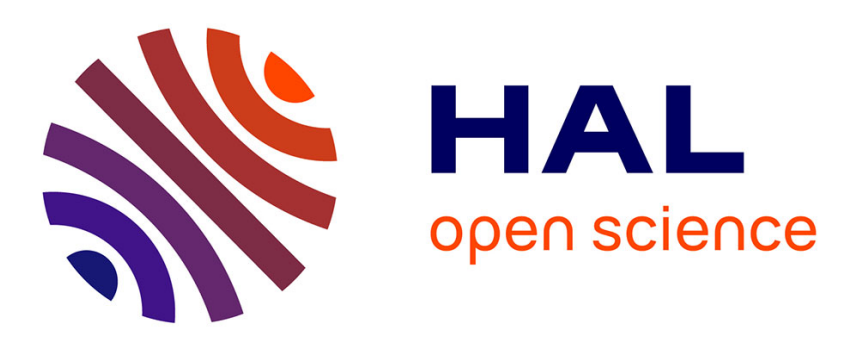

\title{
Mettre en ouvre la transition agroécologique: une analyse des règles de décision dans les systèmes bananiers aux Antilles françaises
}

\author{
Valérie Angeon, Samuel Bates
}

\section{- To cite this version:}

Valérie Angeon, Samuel Bates. Mettre en œuvre la transition agroécologique : une analyse des règles de décision dans les systèmes bananiers aux Antilles françaises. Revue d'Économie Régionale \& Urbaine, 2020, Mars (3), pp.503-529. 10.3917/reru.203.0503 . hal-03135324

\section{HAL Id: hal-03135324 \\ https://hal.inrae.fr/hal-03135324}

Submitted on 8 Feb 2021

HAL is a multi-disciplinary open access archive for the deposit and dissemination of scientific research documents, whether they are published or not. The documents may come from teaching and research institutions in France or abroad, or from public or private research centers.
L'archive ouverte pluridisciplinaire $\mathbf{H A L}$, est destinée au dépôt et à la diffusion de documents scientifiques de niveau recherche, publiés ou non, émanant des établissements d'enseignement et de recherche français ou étrangers, des laboratoires publics ou privés. 


\title{
Mettre en œuvre la transition agroécologique : une analyse des règles de décision dans les systèmes bananiers aux Antilles françaises
}

\section{Implementing the agroecological transition: an analysis of decision-making rules in banana farming systems in the French West Indies}

\author{
Valérie ANGEON \\ INRAE - UR Ecodéveloppement \\ valerie.angeon@inrae.fr \\ Auteur correspondant
}

Samuel BATES

Université d'Angers - GRANEM

samuel.bates@univ-angers.fr

Mots-clés : exploitation agricole, règle de décision, système dynamique, transition agroécologique, verrouillage, viabilité.

Keywords : farming system, decision-making rule, dynamic system, agroecological transition, lock in, viability.

Classification JEL : C60, O13, O33, N66. 


\section{Résumé}

Cet article porte sur la transition agroécologique des exploitations agricoles aux Antilles françaises. Il vise à déterminer les règles de décision à observer dès aujourd'hui afin d'assurer une évolution viable de ces systèmes de production. Un modèle de viabilité avec cible (atteindre un seuil élevé de qualité de sol) est proposé. Les résultats du modèle permettent de définir en combien de temps, à quel coût et selon quelles modalités de pratiques et de choix de production, une exploitation peut opérer sa transition agroécologique. Le modèle est appliqué aux exploitations bananières appréhendées comme des agroécosystèmes. Il permet de sélectionner les options de décision susceptibles d'orienter la trajectoire d'évolution de ces systèmes dynamiques dans le sens souhaité.

\section{Abstract}

This article deals with the implementation of the agroecological transition in the French West Indies. To address this challenge, a viability model is suggested. This approach offers an original framework to highlight agroecosystems dynamics by allowing consideration of simultaneously economic and agronomic dimensions. This holistic approach to agroecosystems is designed at the farming system scale. Farming systems, which are thought as socio-ecosystems (i.e., ecological systems inextricably connected to a socio-economic matrix), represent an essential component of the agricultural sector. Farming systems are a strategic level on which to tackle the different components of viability from a holistic point of view. Farming systems are at the crossroads of agro-technical, organizational, institutional and territorial innovations. They also constitute a consistent level on which to address the issues of complexity and uncertainty. A viability model with target (reaching a high soil quality threshold) is designed, tested and supports operational objectives. The viability analysis enables to answer different questions relating to a given state (i.e. initial state) concerning possible futures. It aims to reveal the set of decision-making rules to be followed to ensure a viable evolution of farming systems. The viability model is applied for banana farming systems. The model results allow to define to what extent a farm can undertake its agroecological transition. It helps to assess the costs and duration of the agroecological transition considering farmers' choices (practices and crops). It shows that postponing the implementation of viable decision rules is costly. The more farmers postpone, the longer it will take for their system to recover viable properties and the more it will cost.

\section{Points-clés}

- Des perspectives de transition agroécologique (TAE) aux Antilles françaises sont mises à jour.

- L'analyse porte sur les systèmes de production bananiers, représentatifs du régime sociotechnique dominant.

- La question de la TAE est posée à l'échelle de l'exploitation agricole.

- Un modèle de décision permet d'anticiper les scénarios d'évolution préservant la viabilité des exploitations.

- Une évaluation de la faisabilité et des coûts de la TAE est proposée compte tenu de l'état initial de l'exploitation. 


\section{-1 - \\ Introduction}

Aux Antilles françaises, l'activité agricole est marquée par une forte spécialisation bananière. Cette orientation productive s'est affirmée, dans les années 1960, suivant la logique de modernisation de l'agriculture basée sur la sélection génétique, l'utilisation d'intrants chimiques de synthèse et de capital fixe (matériel, équipements, bâtiments) pour augmenter la production, les rendements et les revenus.

Cette orientation inscrite dans le paradigme productiviste a généré de nombreux impacts négatifs sur les plans environnementaux et économiques, menaçant la viabilité de l'activité agricole : épuisement des sols, pollution, perte d'efficacité des variétés résistantes aux bioagresseurs, érosion de la biodiversité, dépendance vis-àvis de l'extérieur, déconnexion entre les aires de production et de consommation, renchérissement du coût de l'alimentation, etc. Cet état de fait rend nécessaire la modernisation écologique de l'agriculture. Il s'agit alors de mettre en œuvre la transition agroécologique (TAE) dans ces territoires.

Le changement à promouvoir se pose en termes de conduite de systèmes de production vertueux, respectueux des écosystèmes et du bien-être des populations qui en dépendent. Ce changement est pluriel et tient dans la mise en œuvre d'alternatives, qu'il s'agisse de techniques, pratiques, technologies, choix de production, de commercialisation, etc. Nous faisons l'hypothèse que l'échelle de l'exploitation agricole est pertinente pour le développement d'innovations agrotechniques, organisationnelles, institutionnelles et territoriales susceptibles d'accompagner ces changements. L'exploitation agricole est en effet une unité biophysique et décisionnelle cohérente. En tant qu'écosystème cultivé, une exploitation agricole peut être assimilée à un système écologique et social. Ces écosystèmes cultivés évoluent sous l'effet de l'action humaine. Lieux d'innovations sociotechniques, ils sont pilotés au cours du temps sous contraintes maîtrisables (liées aux choix de production par exemple) ou non (liées aux aléas). Les modalités de gestion établies par l'agriculteur influent alors sur la viabilité de son exploitation. Elles sont révélatrices du rapport qu'entretient l'exploitant avec le vivant.

Nous nous interrogeons sur l'impact des choix de production et de pratiques expérimentés dans les exploitations bananières en Guadeloupe et en Martinique et sur les stratégies à développer pour garantir leur viabilité. Nous cherchons alors à analyser les conditions selon lesquelles les exploitations bananières peuvent envisager une transition vers des systèmes de production plus respectueux de l'environnement, tout en permettant aux agriculteurs d'obtenir un niveau de revenu satisfaisant. Autrement dit, la TAE des exploitations bananières est-elle possible? Si oui, en combien de temps ? À quel coût ? Et selon quelles modalités (avec quels choix de production et de pratiques) ? Il s'agit alors de déterminer les règles de décision qui garantissent la viabilité de ces systèmes de production au cours du temps.

Pour répondre à ces questions, un modèle de prise de décision est élaboré. L'originalité de l'approche est le recours à la théorie mathématique de la viabilité 
qui permet de sélectionner les options de décision à prendre dès aujourd'hui pour orienter l'évolution de systèmes dynamiques (ici un agroécosystème) dans le sens souhaité.

Cet article se décline en trois sections. La section 2 explicite, en mobilisant le courant des sustainability transitions, en quoi l'échelle de l'exploitation agricole est pertinente pour expérimenter et mettre en ouvre la TAE. La section 3 présente le modèle de viabilité proposé. La section 4 est une illustration empirique du modèle qui discute les résultats obtenus dans le contexte agricole des Antilles françaises et formalise les enjeux de la TAE pour les exploitations de ces territoires.

\section{- 2 -}

\section{Raisonner la transition agroécologique à l'échelle de l'exploitation}

La TAE fait l'objet d'une attention croissante dans la littérature et renvoie à un cadre $\mathrm{d}^{\prime}$ analyse fécond qui puise ses origines dans l'approche des transitions durables (sustainability transitions). Parmi les principaux cadres d'analyse qui alimentent ce corpus théorique, figurent ceux des systèmes sociotechniques et des systèmes écologiques et sociaux (Ollivier et al., 2018). Les défis posés par la durabilité des systèmes écologiques et sociaux (McGinnis et Ostrom, 2014) et la mise en œuvre de la TAE imposent de raisonner en considérant les acteurs et les échelles impliqués dans ces processus (Pigford et al., 2018).

\subsection{Légitimité d'un raisonnement à l'échelle de l'exploitation}

L'une des approches les plus usitées de la transition est celle de la multi-level perspective (MLP) (Geels, 2002; 2011) qui propose une analyse des systèmes sociotechniques. En filiation avec les théories économiques évolutionnaires ${ }^{1}$ (Cimoli et Dosi, 1995 ; Arena et Lazaric, 2003 ; Dosi et Winter, 2003), la MLP a pour objet d'analyse le changement. Elle appréhende la transition comme un processus multi-scalaire, multi-acteurs et co-évolutif au sein duquel émergent et se diffusent des innovations sociotechniques motrices du changement. La MLP identifie trois niveaux d'analyse ("paysage sociotechnique ", " régime sociotechnique » et " niche d'innovations ") à partir desquels s'expliquent les dynamiques de changement. Le paysage se situe à l'échelle macroéconomique, le régime à l'échelle mésoéconomique et les niches à l'échelle microéconomique.

Parmi ces trois niveaux, le régime est un point d'attention central puisque c'est en réalité lui qui est amené à faire transition. Les trois niveaux évoluent à des rythmes différents : contrairement à la niche qui est fortement évolutive, le paysage est considéré comme une structure inertielle. Le régime, quant à lui, peut se transformer sur des horizons plus ou moins longs et de manière incrémentale ou radicale en fonction des pressions exercées par le paysage et la niche. Le régime résiste ou s'adapte à ces pressions. Il connaît une temporalité par à-coups. 
Un régime sociotechnique est un ensemble d'acteurs organisés autour de technologies, de ressources, d'infrastructures et dont les relations sont intermédiées par des règles instituées. Ces acteurs sont pluriels relevant des mondes industriel, marchand, politique, scientifique, culturel, etc. Ils sont au fondement de modèles de production et de consommation relativement stables. Pour ce qui concerne la TAE, le changement dont il est question est celui du passage d'un modèle de production et de consommation, fondé sur le paradigme productiviste encadrant et impulsant des innovations techno-productives, à un modèle alternatif promouvant la modernisation écologique de l'agriculture et recourant à des solutions technologiques fondées sur la nature.

La notion de paysage sociotechnique désigne les macro-institutions (paradigmes, aspirations sociétales) et macro-événements (chocs ou autres perturbations naturelles et/ou sociales) qui chapeautent le régime. Le paysage fournit ainsi des signaux susceptibles d'orienter le régime. Dans le cadre de la TAE, en réponse aux défis sociétaux majeurs (changement climatique, explosion démographique, épuisement des ressources naturelles, etc.), le paysage sociotechnique affiche un certain nombre de messages visant à " produire autrement " en s'inscrivant dans le paradigme de la révolution doublement verte. Exogène au régime, le paysage est une invitation (voire une injonction) pesant sur le régime pour faire transition. La MLP reconnaît que les pressions exercées par le paysage créent des "fenêtres d'opportunité » qui favorisent l'intégration dans le régime de nouveaux acteurs, technologies, ressources et règles. Toutefois, même lorsque le paysage impose des injonctions au changement, les options concrètes retenues par le régime ne sont pas connues à l'avance ; les directions que suivra le régime ne sont pas prédites par le paysage.

La niche d'innovation est un espace d'incubation au sein duquel sont définies et testées des technologies alternatives radicales. Ces innovations sont portées par des acteurs en marge du régime sociotechnique dominant et qui demeurent souvent épars. Dans le cadre de la TAE, les innovations radicales se caractérisent par une refondation des relations avec la nature et se traduisent, notamment, par la mobilisation/valorisation de la biodiversité dans les systèmes de production. Il s'agit de solutions sociotechniques visant à " faire avec la nature " ou à " faire faire à la nature ».

En tant qu'acteur dont la finalité est de produire et de vendre leurs productions, les exploitations agricoles participent à la formation de systèmes et de modèles de production. Elles sont en cela des acteurs parties prenantes du régime sociotechnique. Les exploitations travaillent en fonction du régime en place mais elles sont aussi des acteurs de la traduction concrète des nouvelles injonctions émises par le paysage. Elles sont au centre de tensions qui s'exercent sur elles, tant sur le plan des grandes aspirations de la société que sur celui des réalités concrètes, techniques et économiques. On peut donc distinguer deux fonctions en partie contradictoires des exploitations, celle d'incarner le régime en place et celle de mettre en œuvre de nouvelles aspirations. 
Acteur d'un régime en place, les exploitations agricoles se soumettent à des règles de fonctionnement stabilisées. Celles-ci sont de trois ordres : cognitives, de régulation et normatives (Audet, 2015). Les règles cognitives désignent les croyances, les systèmes de normes et de représentations qui organisent la dimension technique des manières de produire. Ces routines institutionnalisées définissent un ordre socioéconomique au sein duquel chaque agent possède un rôle bien établi. Par exemple, dans le cadre du régime productiviste, la conception et la réalisation des innovations sont déléguées aux acteurs de la recherche et de l'industrie et leur mise en application aux agriculteurs. Les règles de régulation comprennent l'ensemble des dispositifs qui rendent possibles les rapports de production et d'échange au sein du régime. Elles correspondent aux règles de droit du régime (droit commercial, règles marchandes, règles de propriété intellectuelle, réglementations environnementales, politiques agricoles, etc.). Enfin, les règles normatives portent sur des valeurs socialement partagées telles que les critères de qualité des produits, d'efficacité de la production, de durabilité, etc.

Les exploitations sont également des lieux de conception et/ou de mise en œuvre des innovations. Si dans le cadre du régime productiviste, les agriculteurs sont prioritairement des utilisateurs d'innovations technologiques, dans le contexte de la TAE, leur place dans le processus d'innovation est amenée à évoluer. Est en effet en jeu le passage d'un modèle descendant de transfert de technologie à une approche systémique de l'innovation (Hall, 2005 ; Klerkx et al., 2010) où les agriculteurs sont amenés à (co)concevoir les innovations. Ce changement de posture est d'autant plus encouragé que les innovations relevant de solutions fondées sur la nature augmentent la complexité et l'incertitude des systèmes de production. Ces degrés de complexité et d'incertitude sont liés, d'une part, au fait que le recours aux processus écologiques ne fonctionne pas de manière aussi prédictible que les processus artificialisés de la production intensive, et d'autre part, au caractère incomplet et non stabilisé des connaissances scientifiques sur les processus écologiques impliqués dans la mobilisation des régulations naturelles. Dans ce cas, la procédure de résolution des problèmes fondée sur la relative maîtrise de la nature, à laquelle avaient recours les acteurs dans le cadre du modèle productiviste, est difficilement opérante. L'appareillage de preuves scientifiques et industrielles qui prévalait jusqu'alors est remis en cause et avec lui l'ordre économique et social qui s'appuyait sur une spécialisation des fonctions des acteurs pour la conception, la production et l'utilisation de technologies standardisées et réplicables. Les innovations dont il est question rendent compte du rapport qu'entretient l'agriculteur à la nature. Elles ne sont pas que technologiques mais aussi d'ordre organisationnel, cognitif, etc.

L'exploitation agricole est incluse dans un régime et accueille des niches. C'est un système socioéconomique (au sens où elle est un lieu de décision et de mise en ouvre d'actions) en même temps qu'elle est un lieu de déploiement d'actes sociotechniques et un système socio-écologique puisqu'il s'agit d'écosystèmes cultivés. En cela, une exploitation agricole est un système écologique piloté à l'aide d'artefacts technologiques et/ou d'objets naturels ( $c f$. Figure 1) et joue à ce titre un rôle dans la TAE. Les processus de décision de l'exploitation sont à la fois contraints (par les règles du paysage et du régime) et libres (par les choix propres de l'agriculteur). Ils 
donnent lieu à la coexistence de divers systèmes de production s'inscrivant dans le régime ou restant en marge du régime. Les agriculteurs (porteurs de visions, de choix techniques, de pratiques différenciées) contribuent ainsi à faire évoluer le régime en place. Compte tenu du nombre et de la grande diversité des exploitations, les chemins de transition sont pluriels et non exclusifs.

\section{Figure 1 - L'exploitation agricole : un lieu d'expérimentation et de mise en œuvre de la transition agroécologique}

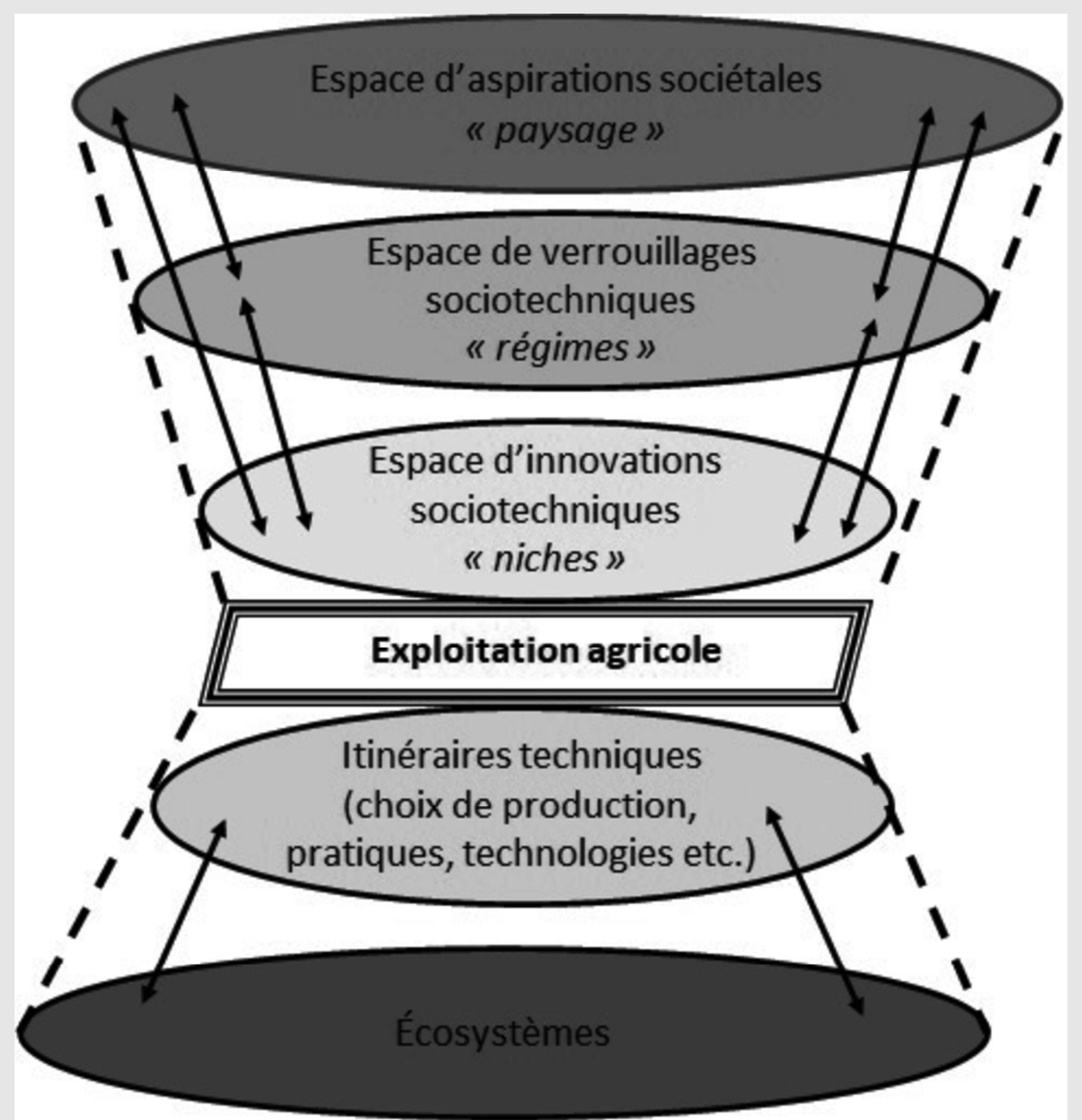

Source : Auteurs.

Pour autant, s'il est vrai que les agriculteurs participent soit à la stabilisation du régime en contribuant à la validation des règles définies aux échelles macro et mésoéconomique, soit à son évolution, l'action d'un agriculteur prise isolément ne peut à elle seule provoquer la transition. C'est très souvent par des collectifs d'acteurs (Kivimaa et Kern, 2016) que s'amorce la transition. La TAE implique de comprendre les ressorts de l'action qui reposent à la fois sur des fondements 
individuels et sociétaux. Cela nécessite de dépasser le dualisme conceptuel entre individualisme et holisme méthodologique (Giddens, 1984) au bénéfice d'une posture holindividualiste (Chanteau, 2003).

Le raisonnement tenu à l'échelle de l'exploitation agricole invite à expliciter la mise en œuvre de la TAE en considérant à la fois les interdépendances entre systèmes social, écologique et sociotechnique et les interactions individuelles et collectives. De ce fait, un raisonnement à l'échelle de l'individu fait sens dans la mesure où l'individu s'inscrit dans un cadre social. Il s'agit d'appréhender la prise de décision de l'agriculteur compte tenu des dynamiques biophysiques et socio-économiques avec lesquelles il doit composer (Schlüter et al., 2017).

\subsection{Légitimité d'un raisonnement en termes de viabilité centré sur l'individu}

L'agriculteur est un acteur décisionnel, c'est-à-dire qu'il opère des actions de contrôle, pour reprendre les usages sémantiques de la théorie de la viabilité (Aubin, 1991, 2010; Aubin et al., 2011). En filiation avec l'analyse intégrée centrée sur l'agent (IAC: Integrated Agent Centred) (Feola et Blinder, 2010), il s'agit d'appréhender les séries de décisions mises en œuvre par les agriculteurs pour piloter les interdépendances (rétroactions dynamiques) s'exerçant entre les sous-composantes (écologiques et sociales) de leurs agroécosystèmes. Parmi ces décisions, nous considérons en particulier le choix des spéculations et des pratiques au sens où elles rendent compte de l'engagement des agriculteurs dans la TAE.

Ainsi, si l'état des agroécosystèmes évolue à travers le temps sous l'effet de facteurs naturels, il est aussi fonction des décisions des agriculteurs. Par ailleurs, l'état du système lui-même influe sur les options de choix possibles revenant à l'agriculteur. Dans ces conditions, les options de décision de l'agriculteur doivent s'adapter chemin faisant à la réalité du système et à la compréhension de leur environnement de production. Ces éléments de compréhension peuvent être imparfaits du fait des capacités cognitives limitées des agents économiques (rationalité limitée). Les options de décision ne peuvent être déterminées à l'avance : elles ne sont ni stables ni statiques. Cela autorise dès lors l'agriculteur à explorer une diversité de solutions de pilotage et à opter pour les plus opportunes compte tenu des contraintes du système. Cela correspond à une interprétation dynamique du processus de prise de décision. Ainsi, le raisonnement de l'agriculteur ne peut se réduire à une solution d'équilibre. Les agriculteurs pourront dès lors privilégier une diversité de scénarios d'évolution de leurs choix technico-économiques. Dans le même ordre d'idées, à données contextuelles identiques (c'est-à-dire pour des exploitations agricoles présentant les mêmes conditions de production), les choix des agriculteurs pourront différer, ce qui renvoie à l'hétérogénéité des comportements individuels et à des modalités d'apprentissage différenciées. De l'agrégation des choix individuels peuvent naître des régularités qui nourrissent l'évolution du régime (niveau supérieur) dans lequel s'insère l'exploitation ${ }^{2}$. L'ensemble de ces prérequis théoriques est conforme au programme de recherche de l'économie évolutionnaire (Dosi et Winter, 2003). Il 
s'agit dès lors de proposer une modélisation de la prise de décision à l'échelle de l'exploitation qui :

- (i) adopte une approche systémique et évolutionnaire ;

- (ii) s'inscrit dans une démarche d'anticipation des états futurs du système à partir de scénarios d'évolution plutôt que de simulation prédictive. Cela suppose d'adopter une lecture inverse du temps (lecture rétrograde) qui garantit de sélectionner les séquences d'actions susceptibles de prévenir tout dommage irréversible du système ; - (iii) articule des enjeux de court terme et de long terme ;

- (iv) constitue une analyse multicritère qui observe les critères de durabilité.

La théorie mathématique de la viabilité (TMV) initiée par Aubin (1991, 2010 ; Aubin et al., 2011) répond à l'intégralité de ces attributs. Elle formalise l'évolution de systèmes complexes. La TMV adossée au calcul numérique permet de déterminer l'ensemble des contraintes initiales à respecter dès aujourd'hui et à travers le temps pour assurer la durabilité de systèmes dynamiques soumis à un contexte complexe lui-même en évolution.

L'approche viabiliste vise à :

- (i) déterminer si à partir d'une situation initiale, il existe au moins une trajectoire d'évolution conforme aux critères de durabilité que l'on cherche à atteindre ;

- (ii) fournir les règles de décision qui vont garantir la viabilité du système. Elle met ainsi l'accent sur l'intérêt à concevoir des interventions humaines en fonction de l'évolution souhaitée de l'état du système.

La TMV emprunte une logique de prise de décision en boucle fermée. Dans ce cas de figure, les décisions de viabilité ne sont pas prises en fonction du temps mais selon l'évolution de l'état du système (à chaque changement d'état du système, on sait quelle règle de décision exécuter quel que soit le moment où intervient cette décision). Le raisonnement en boucle fermée rend possible l'adaptation à temps du système compte tenu de l'évolution de son état et de son contexte d'évolution.

La TMV est une approche directe, inverse et ensembliste. Elle est dite directe car à partir d'un état initial donné auquel on attribue une loi d'évolution, on cherche à vérifier si un état final respectera ou non les propriétés voulues. Elle est inverse ce qui signifie que l'on calcule l'ensemble des états initiaux pour lesquels au moins une des évolutions respectera toujours les propriétés voulues. Elle est ensembliste au sens où il n'existe pas une solution unique mais potentiellement plusieurs chemins de viabilité.

En résumé, la viabilité décrit un état dans lequel il existe au moins une stratégie d'évolution partant d'une position initiale du système qui préserve dans le temps les propriétés nécessaires à la poursuite de son évolution dans des conditions considérées comme souhaitables. Dès lors, un déséquilibre peut être viable (Krawczyk et Kim, 2009 ; Bonneuil et Boucekkine, 2014). 
Pour toutes ces raisons, la TMV est mobilisée pour modéliser les règles de décision dans les systèmes écologiques et sociaux (Mouysset et al., 2013 ; Sabatier et al., 2014 ; Bates et Saint-Pierre, 2018 ; Oubraham et Zaccour, 2018).

\section{- 3 - \\ Les enjeux de la transition agroécologique aux Antilles françaises}

Un modèle permettant d'identifier les choix de spéculations et de pratiques à observer pour conduire la TAE est présenté. Le modèle s'applique à l'échelle de l'exploitation agricole appréhendée comme un système écologique et social où se déploient des innovations sociotechniques dont on cherche à garantir des propriétés de durabilité. Compte tenu de l'importance économique et sociale de la banane aux Antilles françaises, le raisonnement tenu porte sur la capacité des systèmes de production bananiers à s'engager dans la transition.

\subsection{Une logique productive génératrice de mécanismes d'auto-renforcement du modèle conventionnel}

Selon les données fournies par les services déconcentrés du Ministère de l'Agriculture (Ministère de l'Agriculture et de l'Alimentation, 2017), la banane occupe plus de $25 \%$ de la surface agricole utilisée (SAU) à la Martinique et compte pour $54 \%$ de la production agricole totale. Les exploitations bananières présentent une superficie moyenne de 14,4 hectares. En Guadeloupe, la banane occupe $7 \%$ de la SAU et contribue à $10 \%$ de la production agricole totale. La superficie des exploitations bananières est de 6,2 hectares en moyenne. Leur taille est plus élevée que celle des autres productions. Pour autant, le niveau des rendements (moins de 30 t/ha) reste faible en comparaison de celui des principaux pays producteurs de banane (Costa Rica, Guatemala, Côte d'Ivoire) dont les rendements oscillent entre 45 et 50 t/ha (FAOSTAT, 2018).

Tels qu'ils se présentent aux Antilles françaises, les systèmes de production bananiers sont, comme cela est également observé dans d'autres filières agricoles (Fares et al., 2012 ; Meynard et al., 2014 ; Magrini et al., 2016), le résultat de processus de compétition et de sélection technologiques. Le terme " technologie » est entendu ici au sens large. Il décrit aussi bien des objets matériels qu'immatériels (Rip et Kemp, 1998 ) et se rapporte indifféremment à un choix de technique de production, de produit, de norme ou de paradigme (Meynard et al., 2014). Originellement étudiés par Arthur (1989), ces processus de compétition et de sélection technologiques permettent de comprendre par quels mécanismes le choix initial d'une technologie se renforce expliquant alors qu'une technologie dite "inférieure » (sous-efficace) se diffuse et s'impose au point de devenir dominante. 
Plusieurs mécanismes générant des rendements croissants d'adoption sont à l'origine de la compétition technologique. Les rendements croissants d'adoption décrivent une situation selon laquelle l'utilité procurée par l'adoption d'un bien augmente avec son degré de diffusion. Cinq mécanismes sont au fondement des rendements croissants d'adoption: les économies d'échelle en production, la compatibilité technologique, l'apprentissage lié à l'utilisation du produit, les économies de réseau et les rendements informationnels. Chacun de ces mécanismes renforce l'existence de la technologie dominante, soit qu'elle est produite et diffusée à grande échelle et à moindres coûts (économies d'échelle), consolidée à travers un paquet technologique (compatibilité technologique), appropriée à travers son utilisation (apprentissage lié à l'usage), privilégiée du fait que sa communauté d'usagers soit importante (économies de réseau), un bon révélateur d'informations qui tend à augmenter la sphère d'adoptants (rendements informationnels).

La trajectoire des exploitations agricoles aux Antilles françaises est déterminée par le poids et l'enchevêtrement des mécanismes d'auto-renforcement cités précédemment. En effet, l'agriculteur se retrouve enfermé dans un processus de simplification de son système de culture (monoproduction) impliquant la concentration des moyens de production pour augmenter les rendements tout en réduisant les coûts (économies d'échelle). De ce point de vue, l'utilisation d'intrants de synthèse et la sélection variétale constitue un paquet de technologies compatibles. Ce paquet technologique est une condition de robustesse de ces systèmes de culture simplifiés. Ces derniers doivent être en effet alimentés par des ressources extérieures, le milieu naturel n'étant plus en mesure de leur fournir des apports satisfaisants pour un niveau de rendement défini comme acceptable.

Largement diffusé par l'organisation professionnelle des producteurs ("groupement »), le paquet technologique proposé en milieu bananier est utilisé par la majorité des agriculteurs. Son utilisation à grande échelle crée des routines et cristallise des pratiques qui confortent la technologie privilégiée (économies de réseau). On souligne également le fait que les agriculteurs œuvrant dans ces systèmes de production sont en réalité de simples utilisateurs du paquet technologique conventionnel (learning by using) selon l'ordre socioéconomique établi. Ils ratifient et stabilisent ainsi les règles cognitives qui organisent la dimension technique des manières de produire.

Myopes à l'état des connaissances disponibles, les agriculteurs ancrent leur croyance dans le fait que la technologie dominante est soit la seule à exister, soit la meilleure (rendements informationnels). Ces règles normatives sont socialement partagées et la technologie dominante impose ses critères de standardisation des produits, d'efficacité de la production, de commercialisation etc. Sur la base d'un comportement mimétique, les agriculteurs expriment leur préférence pour la technologie dominante, ce qui verrouille in fine le régime sociotechnique dominant.

La persistance de la technologie dominante est d'autant plus affirmée que les acteurs de la filière banane activent différents dispositifs qui entretiennent les rendements croissants d'adoption. Parmi ces dispositifs, les mesures de sécurisation 
des revenus jouent un rôle central, qu'il s'agisse de crédits bancaires, d'octroi d'aides ou de règles d'accès au marché européen. Ces règles de régulation normalisent les rapports de production et d'échange au sein du régime. Dans un contexte de rationnement du crédit bancaire, de concurrence entre filières pour capter les subventions européennes, de complémentarité entre financements publics et privés au profit des producteurs bananiers conventionnels, ces dispositifs attisent des inerties de comportement et de choix des agriculteurs.

Dans ce contexte, les innovations qui contestent la technologie dominante peinent à émerger. Ces dispositifs qui président au processus de sélection compétitive verrouillent le système conventionnel qui s'inscrit dans un sentier de dépendance (path dependency). Il s'agit dès lors de concevoir comment les agriculteurs peuvent s'affranchir du régime sociotechnique dominant, en rendant compatibles des objectifs de performances économique et environnementale. Cette question de la double performance (Guillou et al., 2013) est reformulée à travers les enjeux de la TAE. Il s'agit de promouvoir la durabilité des systèmes de production agricoles. Un tel objectif nécessite que les exploitations agricoles soient viables, c'est-à-dire qu'elles parviennent à préserver leur capital naturel tout en dégageant un niveau de revenu satisfaisant. En ce sens, la viabilité des exploitations agricoles s'inscrit en filiation du paradigme de la durabilité forte (Durand et al., 2012).

\subsection{Modélisation de la transition agroécologique en termes de viabilité}

S'agissant d'entreprendre la TAE, diverses voies peuvent être explorées. Le spectre des chemins de transition oscille entre deux stratégies extrêmes : écologiser les systèmes de production de bananiers existants et développer des systèmes de production alternatifs à la banane. Considérant le poids prépondérant des monocultures d'exportation ainsi que tous les verrous du régime sociotechnique, nous nous demandons dans quelle mesure la TAE peut être conduite à partir des exploitations agricoles spécialisées en bananes. À quel coût et en combien de temps peut-on, à partir d'une situation de production initiale caractérisée par une monoculture d'exportation, atteindre un objectif fixé de double performance économique et environnementale? La formalisation de ces questions et leur ensemble de solutions sont proposées à travers un modèle de viabilité.

Est considérée ici comme viable toute exploitation bananière qui a la possibilité d'atteindre ou de rester dans un état défini comme souhaitable tout en respectant à chaque moment des contraintes que l'on se donne portant sur la qualité du sol et sur le revenu. Ainsi, peut-on assurer qu'à partir d'une situation initiale (systèmes de culture bananiers intensifs), des objectifs de performance environnementale et économique soient atteints? Il s'agit de déterminer des choix de spéculations et de pratiques agricoles permettant de préserver et/ou de restaurer la qualité du sol tout en assurant à l'agriculteur un revenu satisfaisant. 
Un portefeuille de couples spéculations-pratiques alternatifs aux systèmes bananiers conduits de manière conventionnelle est mobilisable par l'agriculteur. L'exploration de cette diversité de choix suppose des capacités de flexibilité de la part de l'agriculteur pour passer à travers le temps d'une spéculation à une autre et d'une pratique à une autre. Une telle hypothèse est envisageable uniquement si l'agriculteur possède les compétences requises pour effectuer ces changements de spéculations et de pratique au fil du temps.

Afin de respecter un degré de réalisme, nous considérons un portefeuille de spéculations réduit. Il se compose de spéculations semi-pérennes (cinq ans) : la banane d'export, la banane plantain (dédiée au marché local), la canne à sucre. À ceci s'ajoute la jachère courte et la jachère pâturée (pâturage bovin). Ces spéculations présentent des propriétés en termes de restauration/préservation des sols (biomasse produite). Par ailleurs, ces choix de spéculations sont compatibles en termes de maîtrise technique et sont d'ailleurs observés dans la pratique. Ainsi, l'agriculteur dont la compétence est exclusivement bananière à la date initiale pourra au fil du temps changer de spéculation sans supporter de coûts d'apprentissage.

Deux modes de production sont considérés. Ils correspondent à un éventail de pratiques conventionnelles (paquet technologique productiviste) ou agroécologiques (solutions technologiques fondées sur la nature). Chacune des spéculations décidées par l'agriculteur peut par conséquent être conduite selon des pratiques conventionnelles ou agroécologiques. Les choix de pratiques privilégiés influent sur l'état du sol, en particulier sur sa qualité biologique (densité et diversité d'organismes vivants présents dans le sol).

Le modèle de viabilité élaboré trouve ses origines dans Durand et al. (2017). La dynamique de l'exploitation est décrite par les variables d'état (qualité de sol et niveau de revenu) qui rétroagissent et qui évoluent en fonction de variables de contrôle (choix de couple spéculation-pratique), sachant que ce qui se produit à la période $n+1$ dépend de ce qui s'est produit à la période $n$ ( $c f$. Figure 2).

Les choix de spéculations et de pratiques permettent à l'agriculteur initialement spécialisé dans la production bananière de modifier ses arbitrages entre performances économique et environnementale au cours du temps. Cet arbitrage est réalisable sur un intervalle de temps fini ( $T=40$ ans) qui correspond à une durée d'activité professionnelle complète (période d'activité) exercée par l'agriculteur sur une même exploitation. Plus la date de transmission de l'exploitation approche, plus l'agriculteur perdra en flexibilité de choix de spéculations et de pratiques pour ajuster à temps ses règles de décision.

$T \leqslant 40$ ans est une contrainte temporelle de cession d'activité et de transmission de l'exploitation. Céder une exploitation revient à transmettre une valeur patrimoniale. La valeur de ce patrimoine est d'autant plus élevée que la santé économique et environnementale de l'exploitation est bonne. 


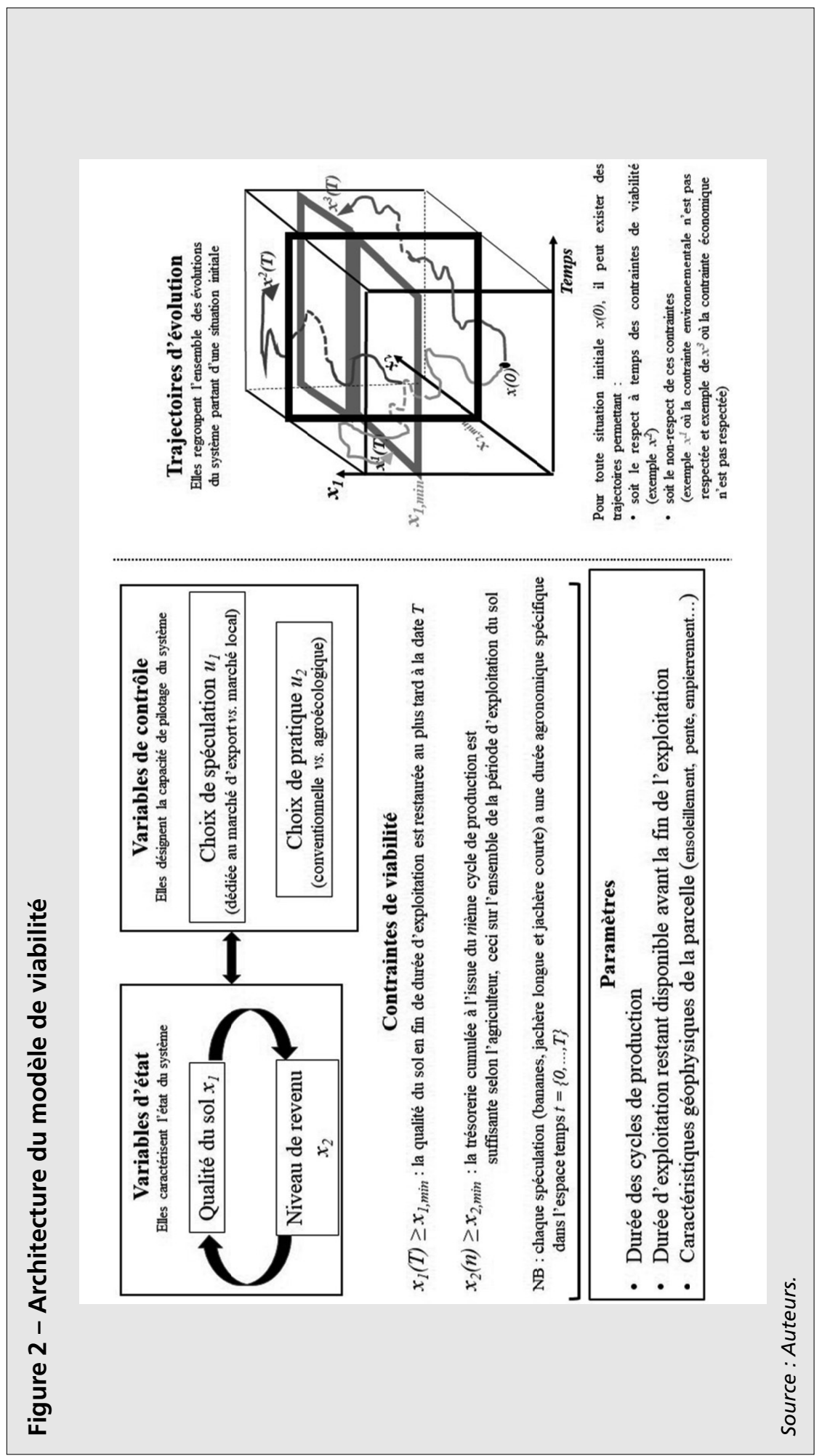


L'évolution des états économique et environnemental de la parcelle de production est régie par le système dynamique $(F)$ composé des trois équations suivantes :

$$
\begin{aligned}
& x 1(n+1)=\Phi(x 1(n), u 1(n), u 2(n)) \\
& x 2(n+1)=x 2(n)+l(x 1(n), u 1(n), u 2(n)) \\
& \tau(n+1)=\tau(n)+\delta(u 1(n), u 2(n))
\end{aligned}
$$

L'Équation 1 traduit l'évolution de l'état du sol $x_{1}(n)$ à l'issue du nième cycle de production. Le cycle de production définit la durée qui s'écoule entre la mise en culture d'une spéculation et la vente de la production obtenue. La variable indicatrice de l'état du sol est un proxy de l'Indicateur Biologique de Qualité du Sol (IBQS) inspiré de Velasquez et al. (2007) et Ruiz et al. (2011). Il s'agit d'un indicateur composite de 54 variables qui relie l'état de la macrofaune (densité, diversité d'espèces indicatrices) aux composantes physiques, chimiques, biologiques des sols. L'IBQS dépend des cultures et des pratiques mises en œuvre et influe sur les rendements. Il est normé sur une échelle de 0 à 1 . Plus il est proche de 1 , plus la qualité du sol héritée des successions culturales et de pratiques passées est bonne. Inversement, plus il est proche de 0, plus la qualité du sol est mauvaise.

L'Équation 2 traduit l'évolution de la performance économique tirée du nième cycle de production. La variable indicatrice de l'état économique du système est saisie à travers la trésorerie cumulée à l'issue du nième cycle de production $x_{2}(n)$.

L'Équation 3 traduit le calendrier des cycles de production. $\tau(n)$ est la date de démarrage du nième cycle de production. $\delta$ est la durée agronomique du nième cycle de production. Cette durée dépend de la spéculation et de la pratique (mode de production conventionnel versus agroécologique) choisies. Le mois est la plus petite unité de temps retenue et ce dans un souci de réalisme puisque la trésorerie est raisonnée mensuellement par les agriculteurs.

L'usage agricole du sol est contrôlé à partir d'un choix de spéculations $u_{1}$ (choix entre cinq spéculations) et de pratiques $u_{2}$. La production de chacune des spéculations du vecteur $u_{1}$ peut être réalisée sous deux modalités choisies par l'agriculteur dans le vecteur $u_{2}$ : pratique conventionnelle versus agroécologique. Chaque spéculation associée à une pratique est conduite selon un itinéraire technique. La conduite des systèmes de culture est entre autres sensible à des paramètres liés aux coûts de production et aux caractéristiques physiques (ensoleillement, pente, empierrement, etc.) et géographiques de l'exploitation (localisation de l'exploitation et proximité à $\mathrm{d}^{\prime}$ autres systèmes de culture conduits de manière conventionnelle ou agroécologique). Ces paramètres sont inclus dans la fonction de variation de revenu $l$ et dans la fonction de variation de la qualité du sol $\Phi$.

L'état de viabilité d'une exploitation agricole est défini en fonction de deux contraintes principales: 
- (i) contrainte économique de court terme : $x_{2}(n) \geqslant x_{2 \min }$. Cette contrainte portant sur la trésorerie cumulée doit être respectée à l'issue de chaque nième cycle de production. Le niveau de $x_{2 \min }$ est spécifique à chaque agriculteur. Il s'agit alors de déterminer les seuils au-dessus desquels tout niveau de trésorerie est dès aujourd'hui jugé satisfaisant par l'agriculteur pour assurer la performance économique et environnementale de son exploitation. Si $x_{2 \min }>0$, alors l'exploitant s'impose de dégager une marge d'autofinancement à chaque fin de cycle de production. Si $x_{2 \min }<0$, alors l'agriculteur pourra s'autoriser une trésorerie négative sur le cycle de production considéré.

- (ii) contrainte environnementale de long terme : $x 1(T) \geqslant x_{1 \min }$. La contrainte environnementale est " terminale " (Aubin et al., 2011) dans la mesure où l'agriculteur n'est pas tenu de la respecter à l'issue de chaque cycle de production. L'hypothèse retenue ici est celle d'une restauration de la qualité du sol avant la fin de la période d'activité (horizon au plus de 40 ans).

On note $K$ l'ensemble des contraintes de viabilité :

$$
\begin{aligned}
& x_{1}(T)-x_{1 \min } \geqslant 0 \\
& x_{2}(T)-x_{2 \min } \geqslant 0
\end{aligned}
$$

compte tenu du temps restant à courir sur l'horizon de 40 ans au plus et sachant la durée incompressible des cycles de production, on a ainsi :

$$
\mathrm{K}=\left([0,1] \times\left[\mathrm{x}_{2 \min },+\infty\right] \times[0, \mathrm{~T}]\right)
$$

Les choix de spéculations et de pratiques expliquent la dynamique d'évolution de l'exploitation. Les conditions de reproduction de l'exploitation au cours du temps impliquent que des choix générant des irréversibilités économiques et/ou environnementales soient évités. Les irréversibilités se manifestent par toute sortie définitive de l'ensemble $K$. L'enjeu est alors de déterminer pour chaque agriculteur souhaitant s'engager dans la TAE, les dates butoirs auxquels les changements de contrôles (couples spéculation-pratique) doivent être opérés.

L'ensemble des états initiaux à partir desquels il existe au moins une trajectoire viable constitue l'ensemble de viabilité (ViabK). En notant $x$ le vecteur de variables d'état et $u$ le vecteur de variables de contrôle, on a :

$$
\operatorname{ViabK}=\left\{\begin{array}{c}
x(0) \mid \exists u \text { tel que }(x(\cdot), u(\cdot)) K, \forall n=1, \ldots, \text { Nk et } \\
\forall t>0 \text { avec } x(n) \text { solution de } x(n+1)=F(x(n), u(n))
\end{array}\right\}
$$

où $N_{K}$ représente le nombre total de successions culturales opérées au fil du temps entre la date initiale $t=0$ et la date finale $t=T$ sachant leur durée respective : $x\left(N_{K}\right)$ $=x(T)$. 
Connaître cet ensemble, c'est identifier toutes les options de régulation viables des systèmes de production agricole au regard des contraintes qui ont été définies. Si le noyau de viabilité est plus large qu'un singleton, il devient possible de hiérarchiser les trajectoires (et donc les options de conduite de la TAE) en fonction d'un critère d'optimalité spécifique.

La Figure 3 schématise la logique de modélisation de la dynamique de l'exploitation agricole.

\section{Figure 3 - Dynamique de l'exploitation agricole}

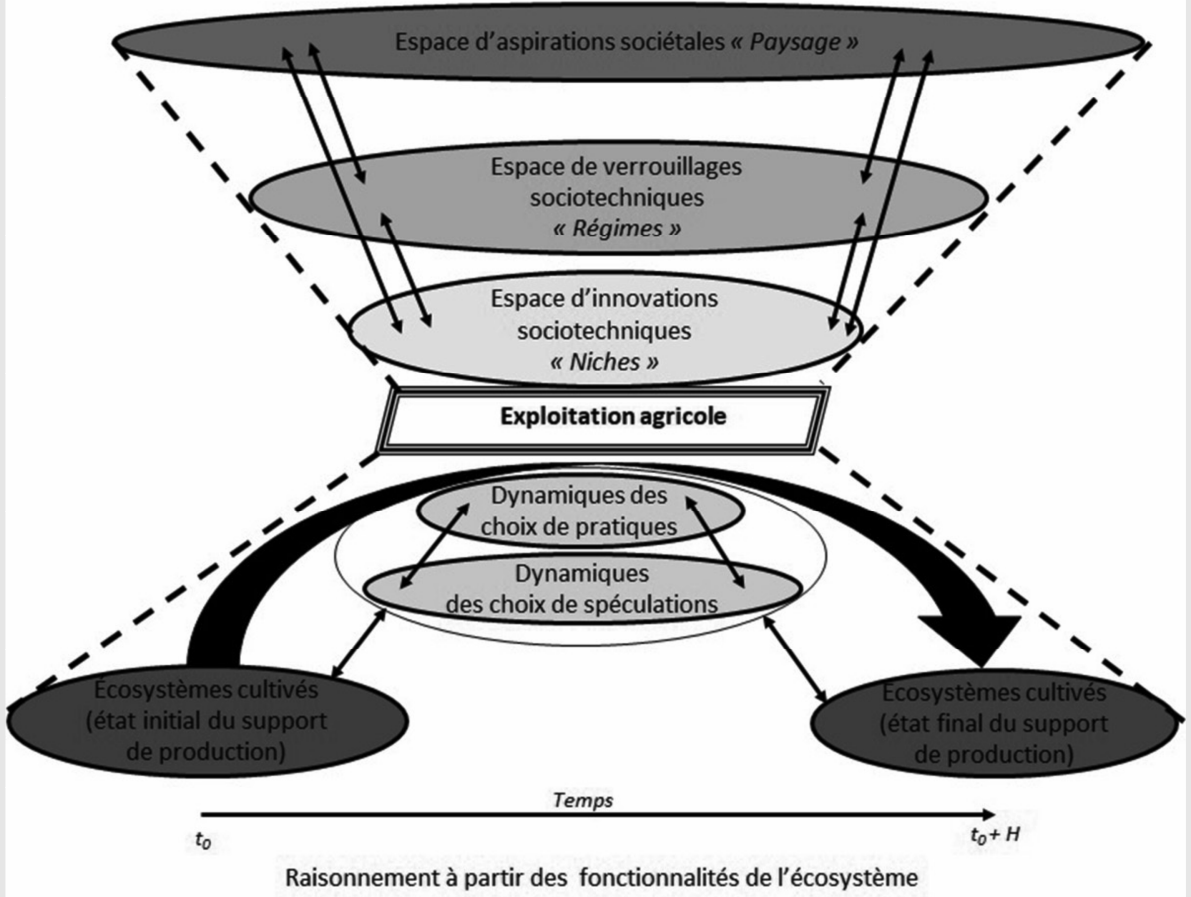

Source : Auteurs.

Dans les illustrations à venir, le critère d'optimalité retenu est celui de la maximisation du revenu. On considère alors que c'est un critère économique de court terme qui prévaut dans la prise de décision de l'exploitant. La sélection d'une telle option de viabilité tend à passer au second plan les conséquences du choix spéculation-pratique sur la qualité du sol. Pour autant, les couples de spéculationpratique choisis au cours du temps ne doivent pas transgresser les contraintes de l'ensemble $K$ par des dommages irréversibles. Retenir un tel scénario permet d'étudier la faisabilité de la transition même dans une configuration d'inertie des comportements conduisant l'agriculteur à continuer à se conformer aux règles du 
régime dominant en optant dans un premier temps pour la même spéculation et la même pratique (la banane avec mode de conduite conventionnel).

\section{-4 - \\ Résultats et discussion}

Le problème de viabilité à résoudre est un problème de cible à atteindre (obtenir un sol de qualité) sur une période donnée (40 ans au plus) par une succession de décisions dans le temps et dans l'espace relatives aux changements de spéculations et de pratiques. Au regard de notre problématique générale (la TAE des exploitations bananières), nous considérons comme situation initiale celle d'une exploitation spécialisée en banane d'exportation.

Les données rentrées pour le calcul numérique sur le plan économique s'appuient sur le dernier référentiel technico-économique produit par la Chambre d'agriculture de la Guadeloupe pour la période 2012-2016. Les variables économiques sont fournies par hectare et par mois. Il s'agit des coûts fixes, des coûts variables (intrants, coûts de la main-d'œuvre), des subventions à la production, des prix à la tonne. Sur le plan agronomique, l'impact des successions culturales sur la qualité du sol a été renseigné sur la base d'analyse de sol et de dires d'experts ${ }^{3}$.

\subsection{Effet mesuré de la monoculture de banane en conduite conventionnelle sur la qualité du sol}

Le module agronomique du modèle montre qu'en dix ans de culture continue de banane conduite de manière conventionnelle (soit après enchaînement de deux cycles de culture), un sol de qualité maximale $(I B Q S=1)$ subit une dégradation forte.

Plus précisément, $\mathrm{l}^{\prime} \mathrm{IBQS}$ perd $28 \%$ de sa qualité initiale en cinq ans et se dégrade de $50 \%$ au bout de dix ans ( $c f$. Figure 4). Ainsi, la spéculation bananière conduite de manière conventionnelle sur un pas de temps long détériore le sol. Quelles sont alors les conditions de viabilité des systèmes bananiers?

\subsection{Conditions de viabilité des systèmes bananiers}

Les résultats du modèle montrent qu'il existe des noyaux de viabilité qui contiennent plus d'une solution.

Les illustrations de la Figure 5 considèrent que : $0,9 \leqslant x_{1 \min } \leqslant 1$ correspond à une bonne qualité de sol et $T=40$ ans, soit 480 mois maximum. Cinq interprétations majeures sont tirées de la figure ci-dessus :

- 1. Pour éviter une dégradation environnementale forte susceptible de menacer les capacités de production et de reproduction de l'exploitation, la stratégie consistant à enchaîner les cycles de production bananière en mode conventionnel peut être suivie 
Valérie ANGEON, Samuel BATES

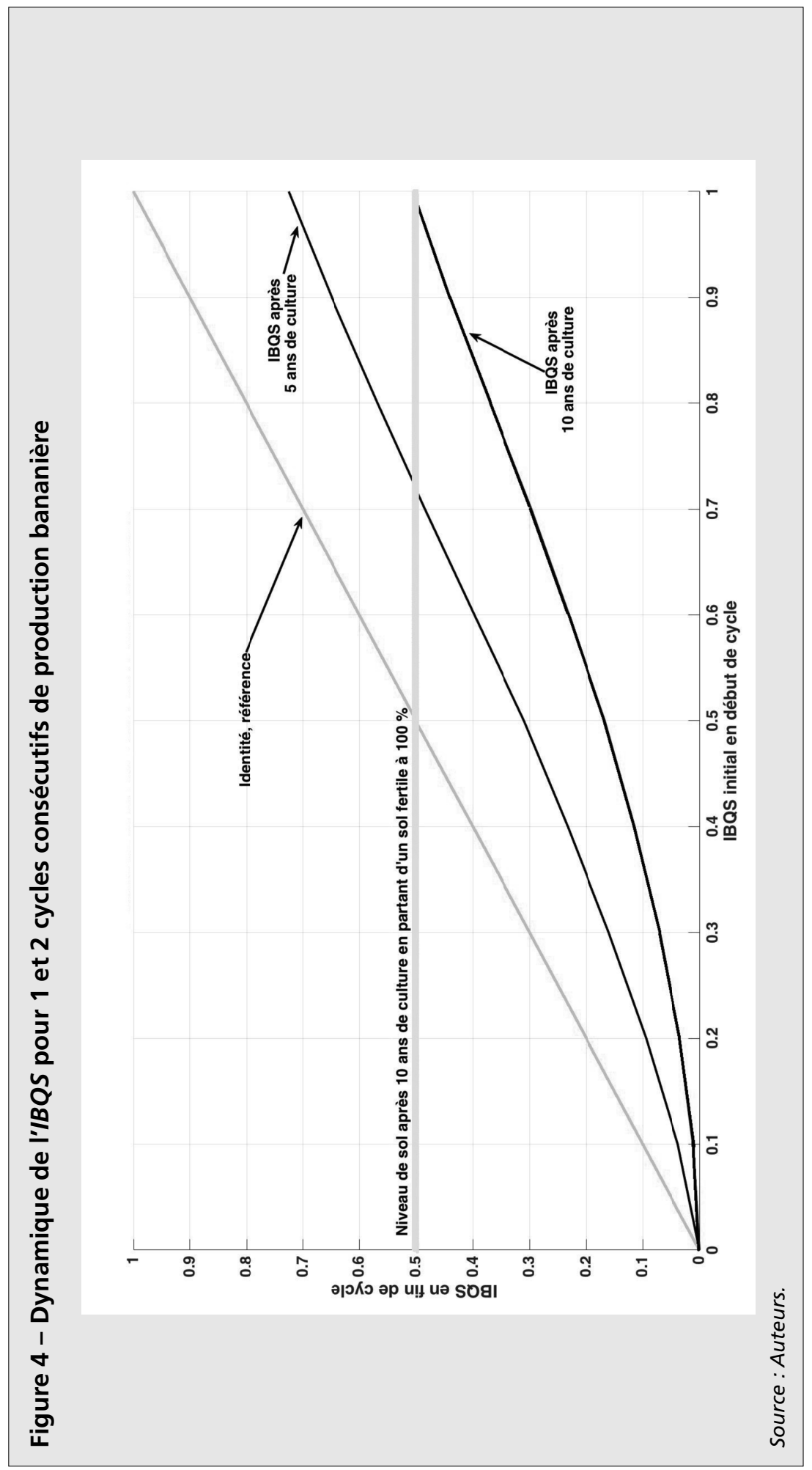




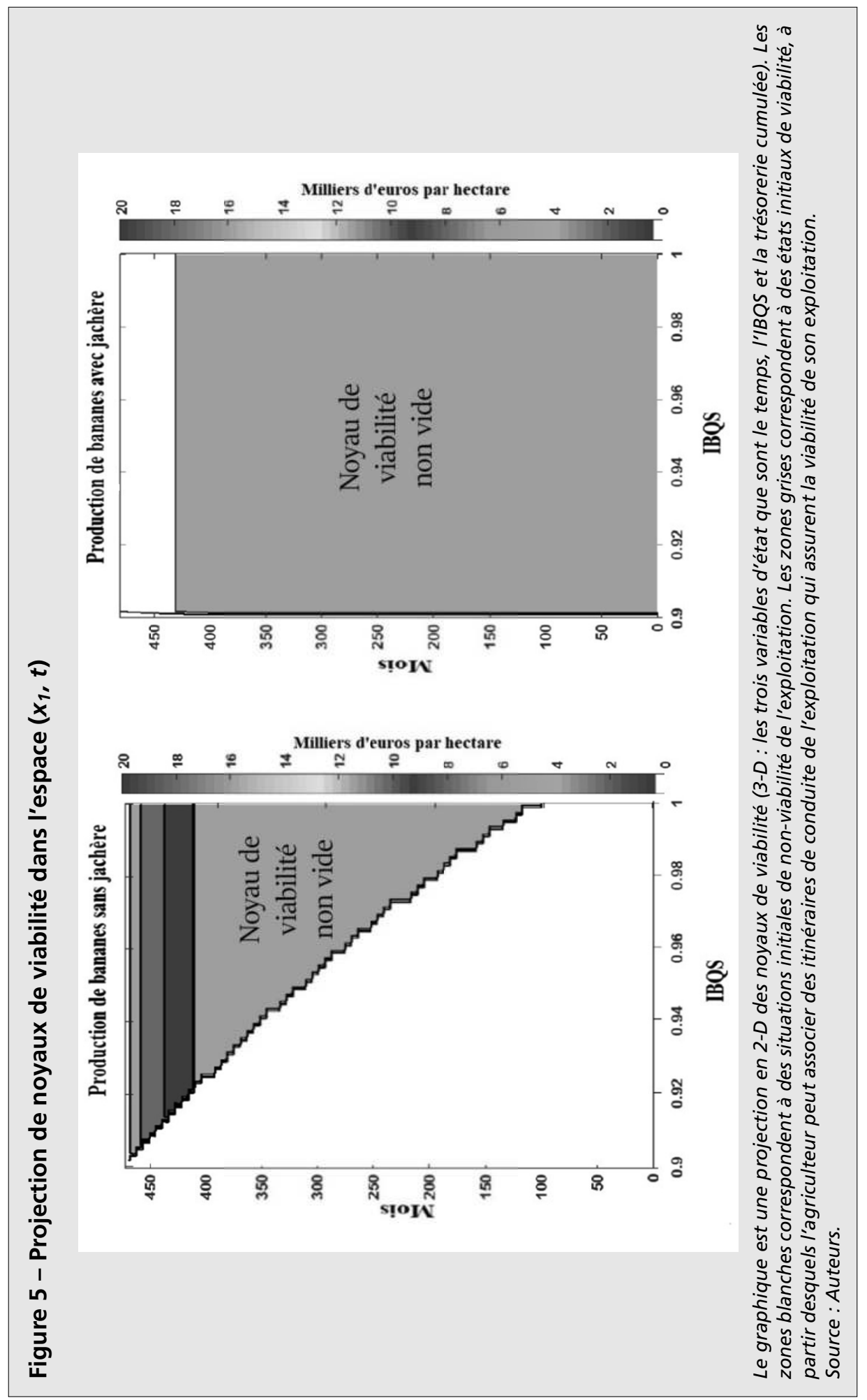


au plus pendant 100 mois et ce pour une excellente qualité initiale de sol. En effet, en partant d'un sol d'une qualité maximale $(I B Q S=1)$, il n'est pas possible pour une exploitation agricole de conserver des propriétés de viabilité au-delà de 100 mois. Il reste au plus 480 - $100=380$ mois à l'agriculteur (soit une trentaine d'années) pour entreprendre une transition vers un autre mode de production (changement à la fois de production et de pratique).

- 2. Pour atteindre l'objectif de double performance, l'agriculteur doit, dans le meilleur des cas, disposer d'au moins 4000 à 6000 euros par hectare d'apport initial. C'est la situation où la qualité initiale de sol est maximale et où le temps d'activité restant est suffisamment long (380 mois).

- 3. L'exploitant peut repousser, dans une certaine limite, ses choix de transition. Au-delà de cette limite, son exploitation perdra ses propriétés de viabilité. Le coût minimal de la viabilité augmente à mesure que la qualité du sol baisse et que le temps d'exploitation restant disponible se réduit. Ce coût peut passer de 4000 euros à au moins 20000 euros par hectare. Cette limite haute correspond au cas où il ne reste que 80 mois d'activité à l'exploitant même avec une excellente qualité initiale de sol $(0,9<I B Q S<1)$. La procrastination augmente le coût de la transition.

- 4. Pour une qualité initiale du sol inférieure $(I B Q S<0,9)$ et pour une durée d'activité productive d'au mieux 380 mois en banane, l'exploitation ne restant qu'en banane conventionnelle ne peut être viable puisque les coûts de transition sont infinis.

- 5. L'acceptation par l'agriculteur d'un temps de restauration de la qualité du sol en recourant à de la jachère pâturée permet d'élargir la taille du noyau de viabilité, en ramenant l'investissement minimal à des niveaux tolérables (4000 à 6000 euros minimum par hectare). Le recours à la jachère pâturée procure ainsi à l'exploitation une forme de robustesse dans sa viabilité.

Les noyaux de viabilité étant non vides, des scénarios de TAE peuvent être discutés.

\subsection{Scénarios de transition agroécologique à partir des systèmes bananiers}

Pour illustrer les conditions de viabilité, nous analysons les trajectoires d'évolution d'exploitations agricoles. Nous présentons les solutions retenues pour deux cas d'étude de qualité initiale de sol variable. Ces exemples extraits des noyaux de viabilité explicitent des choix de spéculations et de pratiques à effectuer pour recouvrer la qualité du sol.

\section{Scénario de TAE pour un sol de qualité initiale moyenne}

Soit le cas d'un producteur de banane dont la qualité initiale de sol est moyenne $(I B Q S=0,5)(c f$. Figure 6$)$. Nous raisonnons ici sur la trajectoire optimale extraite du noyau de viabilité avec comme critère d'optimalité la maximisation de la trésorerie cumulée.

Deux cas de figure sont considérés selon que l'agriculteur supporte ou non une contrainte budgétaire : 
Une analyse des règles de décision dans les systèmes bananiers aux Antilles françaises

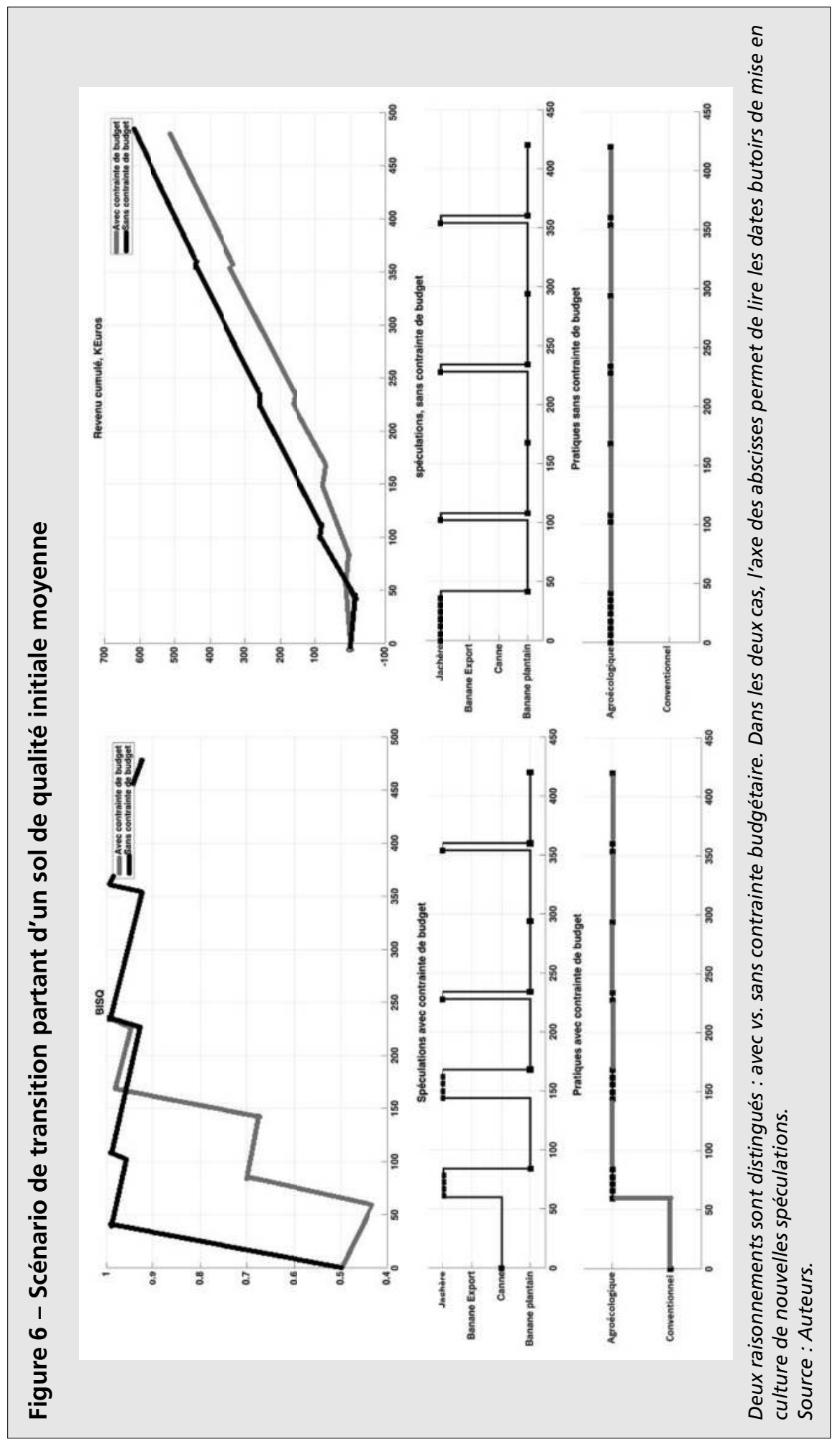


- En l'absence de contrainte budgétaire (c'est-à-dire pour une trésorerie négative tolérable pour l'agriculteur), la stratégie est de privilégier de manière prioritaire et exclusive la restauration du sol. Ainsi, en moins de 50 mois, l'agriculteur restaurera la qualité de son sol jusqu'à atteindre un seuil proche de 1 . Ce scénario de restauration du sol dans un délai réduit passe par un choix de production couplant jachère-banane plantain en conduite agroécologique. La viabilité de l'exploitation agricole nécessite dans ce cas une autorisation de perte financière (revenu cumulé négatif) d'au moins 10000 euros par hectare.

- Sous contrainte budgétaire (impliquant de détenir un revenu cumulé toujours positif), la stratégie consistant à restaurer la qualité du sol prendra au mieux trois fois plus de temps, soit 150 mois. Elle passera par la mise en culture transitoire de canne à sucre avec un mode de conduite conventionnel pour sécuriser le revenu.

\section{Scénario de TAE pour un sol de qualité initiale faible}

Considérons le cas de figure où un agriculteur développe sa production bananière sur 140 mois avec pour conséquence un sol de mauvaise qualité $(I B Q S=0,2)$ (cf. Figure 7). Il ne lui reste que 240 mois au plus pour restaurer son sol.

Pour améliorer la qualité du sol et recouvrer les propriétés de viabilité de son exploitation, l'agriculteur débute par une phase de jachère pâturée au prix d'un effondrement de sa trésorerie cumulée. Il doit ensuite réaliser une production de banane plantain puis de banane d'exportation conduite de manière agroécologique. Il peut ainsi restaurer au final sa trésorerie cumulée (40 000 euros par hectare).

Les deux scénarios étudiés permettent de raisonner sur les conséquences d'un report de transition. Il y a un coût à retarder la mise en place de règles de décision viables. Plus l'agent économique procrastine, plus son exploitation mettra du temps à recouvrer des propriétés de viabilité et plus il lui en coûtera. Le modèle élaboré et les résultats obtenus permettent de déterminer le temps limite et les coûts associés à la mise en ouvre de la transition.

\section{- 5 - \\ Conclusion}

Le paysage agricole des Antilles françaises est marqué par la prédominance de l'agriculture d'exportation. Les exploitations bananières sont les pierres angulaires de ce modèle agro-industriel qui est aujourd'hui largement décrié. Sur cette base, nous nous sommes interrogés sur la conduite de la TAE à partir des exploitations bananières. Pour ce faire, un modèle de viabilité a été conçu. Il détermine en combien de temps et à quel coût une dynamique de changement peut être implémentée à l'échelle de l'exploitation. Cette dynamique de changement exprimée par des choix individuels de spéculation et de pratiques rend compte des innovations à conduire afin d'assurer les conditions de viabilité de l'exploitation. 


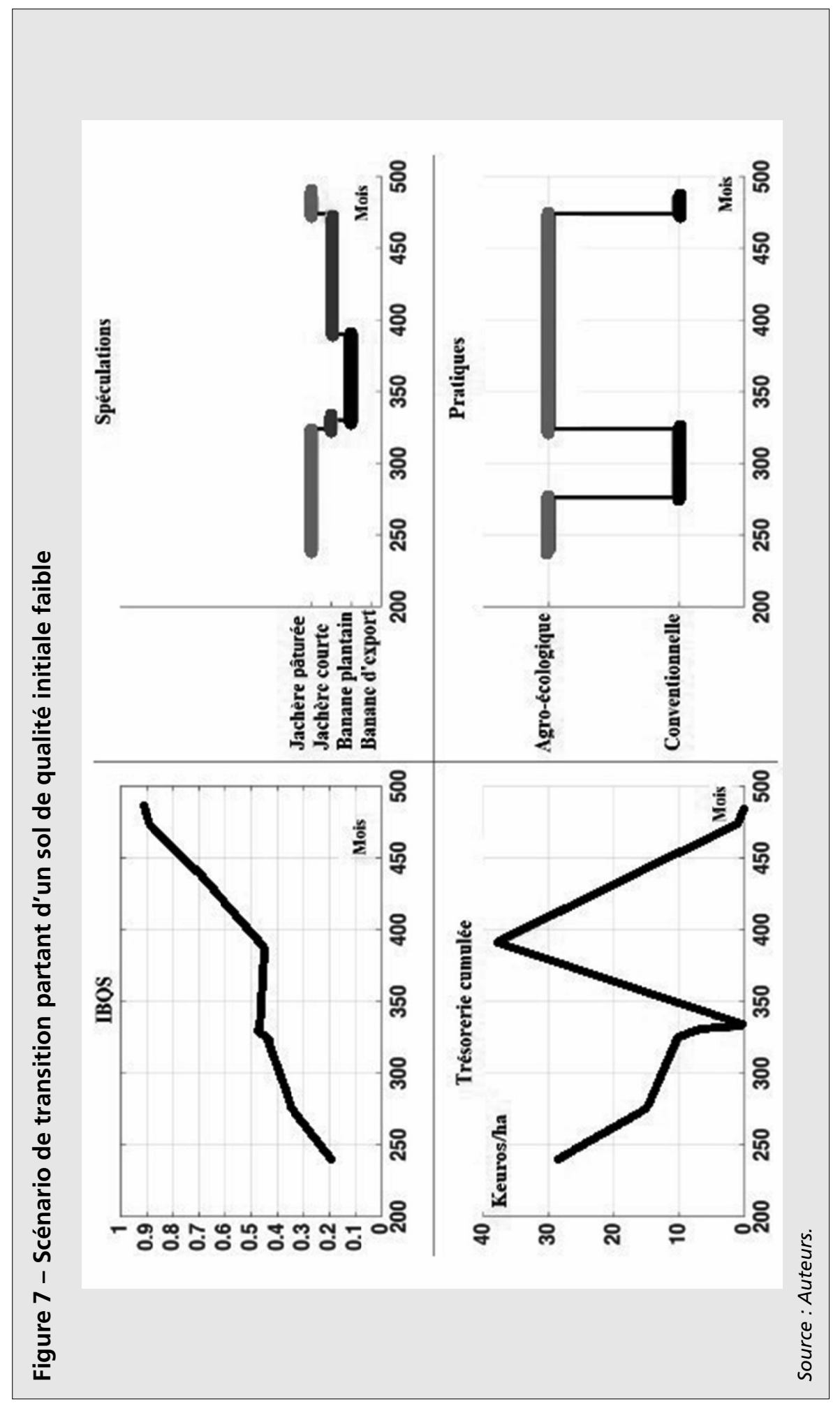


Nos résultats soulignent le poids des contraintes budgétaires. Or les agriculteurs aux Antilles françaises évoluent dans un contexte général de rationnement du crédit comparativement au contexte hexagonal. Dans un tel contexte, la perception des risques par les banquiers privés dessert le financement des innovations (en l'occurrence des systèmes de production alternatifs aux cultures d'export et conduits en pratiques agroécologiques). Ces systèmes de production sont par essence complexes et plus incertains que les systèmes conventionnels inscrits dans le paradigme de maîtrise de la nature. La technicité des processus de financement est d'ailleurs mieux maîtrisée et sécurisée en production conventionnelle notamment du fait des subventions publiques dédiées à ces monocultures historiques et des capacités d'organisation des producteurs. Une transformation des modalités de financement des filières agricoles est un des verrous à lever au sein du régime dominant pour accompagner la TAE.

Le modèle de viabilité élaboré présente deux limites à dépasser pour appréhender de manière fine les conditions de mise en œuvre réelles de la TAE. Premièrement, le modèle est appliqué à des exploitations agricoles mono-parcelles. Considérer une exploitation multi-parcelles permettrait d'introduire dans l'analyse le raisonnement spatial et d'affiner les résultats sur les stratégies de viabilité des agriculteurs. Deuxièmement, le modèle considéré n'intègre pas l'incertitude. Or les exploitations agricoles (et singulièrement celles des Antilles françaises) sont soumises à un certain nombre de variables que les agriculteurs ne contrôlent pas (aléas climatiques, maladies émergentes, fluctuation des prix). Des développements futurs permettront de mieux appréhender les dimensions spatiale et temporelle de la TAE et la robustesse de ces éléments d'analyse en situation d'incertitude.

\section{Remerciements}

Ce travail s'inscrit dans le cadre des projets ANR GAIA-TROP (ANR-12-AGRO0009-05) et AgroEcoDiv avec le soutien financier de l'Europe (FEDER, FSE), de la France et de la Région Guadeloupe. Les auteurs remercient les participants au projet GAIA-TROP et en particulier Anya Désilles ainsi qu'Olivier Crevoisier pour ses relectures et échanges sur ce texte.

\section{Références bibliographiques}

Arena R, Lazaric N (2003) La théorie évolutionniste du changement économique de Nelson et Winter : une analyse économique rétrospective. Revue économique 54 (2) : 329-354.

Arthur B W (1989) Competing technologies, increasing returns, and lock-in by historical events. The Economic Journal 99(394): 116-131.

Aubin J-P (1991) Viability theory. Birkhäuser, Berlin.

Aubin J-P (2010) La mort du devin, l'émergence du démiurge : essai sur la contingence, la viabilité et I'inertie des systèmes. Beauchesnes, Paris.

Aubin J-P, Bayen A M, Saint-Pierre P (2011) A viability theory: new directions. Springer-Verlag Berlin Heidelberg. 
Audet R (2015) Le champ des sustainability transitions : origines, analyses et pratiques de recherche. Cahiers de recherche sociologique 58 : 73-93.

Bates S, Saint-Pierre P (2018) Adaptive policy framework through the lens of the viability theory: a theoretical contribution to sustainability in the Anthropocene Era. Ecological Economics, 145: 244262.

Bonneuil N, Boucekkine R (2014) Viable Ramsey economies. The Canadian Journal of Economics 47(2): 422-441.

Chanteau J (2003) La dimension socio-cognitive des institutions et de la rationalité : éléments pour une approche holindividualiste. L'année de la régulation : Économie, institutions, pouvoirs 6 (2003-2004) : 45-89.

Cimoli M, Dosi G (1995) Technological paradigms, patterns of learning and development: an introductory roadmap. Journal of Evolutionary Economics 5: 243-268.

Dosi G, Winter S G (2003) Interprétation évolutionniste du changement économique : une étude comparative. Revue économique 54 : 385-406.

Durand M-H, Martin S, Saint-Pierre P (2012) Viabilité et développement durable. Natures Sciences Sociétés $20: 271-285$.

Durand M-H, Desilles A, Saint-Pierre P, Angeon V, Ozier-Lafontaine H (2017) Agroecological transition: a viability model to assess soil restoration. Natural Resource Modeling 30 (3).

FAOSTAT (2018) Data [online] http://www.fao.org/faostat/en/\#data.

Fares M, Magrini M-B, Triboulet P (2012) Transition agroécologique, innovation et effets de verrouillage : le rôle de la structure organisationnelle des filières : le cas de la filière blé dur française. Cahiers Agricultures 21 (1) : 34-45.

Feola G, Blinder C R (2010) Towards an improved understanding of farmers' behaviour: the integrative agent-centred (IAC) framework. Ecological Economics 69(12): 2323-2333.

Geels F W (2002) Technological transitions as evolutionary reconfiguration processes: a multi-level perspective and a case-study. Research Policy 31(8-9): 1257-1274.

Geels F W (2011) The multi-level perspective on sustainability transitions: responses to seven criticisms. Environmental Innovation and Societal Transitions 1(1): 24-40.

Giddens A (1984) The constitution of society. Cambridge: Polity.

Guillou M, Guyomard H, Huyghe C, Peyraud J-L (2013) Le projet agro-écologique : vers des agricultures doublement performantes pour concilier compétitivité et respect de l'environnement. Propositions pour le Ministre de l'agriculture, de l'agro-alimentaire et de la forêt, Agreenium/INRA, Paris.

Hall A J (2005) Capacity development for agricultural biotechnology in developing countries: an innovation systems view of what it is and how to develop it. Journal of International Development 17(5): 611-630.

Klerkx L, Aarts N, Leeuwis C (2010) Adaptive management in agricultural innovation systems: the interactions between innovation networks and their environment. Agricultural Systems 103(6): 390400.

Kivimaa P, Kern F (2016) Creative destruction or mere niche support? Innovation policy mixes for sustainability transitions. Research. Policy 45(1): 205-217.

Krawczyk J B, Kim K (2009) Satisficing solutions to monetary policy problem: a viability theory approach. Macroeconomic Dynamics 13(1): 46-80.

Magrini M-B, Anton M, Cholez C, Corre-Hellou G, Duc G, Jeuffroy M-H, Meynard J-M, Pelzer E, Voisin A-S, Walrand S (2016) Why are grain-legumes rarely present in cropping systems despite their environmental and nutritional benefits? Analyzing lock-in in the French agrifood system. Ecological Economics 126: 152-162.

McGinnis M D, Ostrom E (2014) Social-ecological system framework: initial changes and continuing challenges. Ecology and Society 19(2).

Meynard J-M, Messéan A (2014) La diversification des cultures : lever les obstacles agronomiques et économiques. Éditions Quæ, Versailles. 
Ministère de l'Agriculture et de l'Alimentation (2017) Mémento de la statistique agricole. Agreste, DAAF Guadeloupe.

Mouysset L, Doyen L, Jiguet F (2013) From population viability analysis to coviability of farmland biodiversity and agriculture. Conservation Biology 28(1): 187-201.

Ollivier G, Magda D, Mazé A, Plumecocq G, Lamine C (2018) Agroecological transitions: what can sustainability transition frameworks teach us? An ontological and empirical analysis. Ecology and Society 23(2): 219-236.

Oubraham A, Zaccour G (2018) A survey of applications of viability theory to the sustainable exploitation of renewable resources. Ecological Economics 145: 346-367.

Pigford A-A E, Hickey G M, Klerkx L (2018) Beyond agricultural innovation systems? Exploring an agricultural innovation ecosystems approach for niche design and development in sustainability transitions. Agricultural Systems 164: 116-121.

Rip A, Kemp R P M, Kemp R (1998) Technological change. In : Rayner S, Malone E L (eds) Human choice and climate change. Vol II, Resources and Technology. Battelle Press, Columbus: 327-399.

Ruiz N, Mathieu J, Celini L, Rollard C, Hommay G, lorio E, Lavelle P (2011) IBQS: a synthetic index of soil quality based on soil macro-invertebrate communities. Soil Biology and Biochemistry 43(10): 2032-2045.

Sabatier R, Doyen L, Tichit M (2014) Heterogeneity and the trade-off between ecological and productive functions of agro-landscapes: a model of cattle-bird interactions in a grassland agroecosystem. Agricultural Systems 126: 38-49.

Schlüter M, Baeza A, Dressler G, Frank K, Groeneveld J, Jager W, Janssen M A, McAllister R R J, Müller B, Orach K, Schwarz N, Wijermans N (2017) A framework for mapping and comparing behavioural theories in models of social-ecological systems. Ecological Economics 131: 21-35.

Velasquez E, Lavelle P, Andrade M (2007) GISQ, a multifunctional indicator of soil quality. Soil Biology \& Biochemistry 39(12): 3066-3080.

\section{Notes}

1 - Nous optons pour ce terme plus fidèle à la traduction de l'expression anglo-saxonne " evolutionary economics".

2 - Cette remontée (" upscaling ») vers les régimes et le paysage n'est pas explorée dans cet article.

3 - Experts agronomes du centre Antilles-Guyane de l'INRA (aujourd'hui INRAE). 



\section{Note de recherche}

